\title{
UCRL-JRNL-211883
}

LAWRENCE LIVERMORE N A T IO N A L LABORATORY

\section{Generalized in situ adaptive tabulation for constitutive model evaluation in plasticity}

A. Arsenlis, N. Barton, R. Becker, R. Rudd

May 2, 2005

Computer Methods in Applied Mechanics and Engineering 
This document was prepared as an account of work sponsored by an agency of the United States Government. Neither the United States Government nor the University of California nor any of their employees, makes any warranty, express or implied, or assumes any legal liability or responsibility for the accuracy, completeness, or usefulness of any information, apparatus, product, or process disclosed, or represents that its use would not infringe privately owned rights. Reference herein to any specific commercial product, process, or service by trade name, trademark, manufacturer, or otherwise, does not necessarily constitute or imply its endorsement, recommendation, or favoring by the United States Government or the University of California. The views and opinions of authors expressed herein do not necessarily state or reflect those of the United States Government or the University of California, and shall not be used for advertising or product endorsement purposes. 


\title{
Generalized in situ adaptive tabulation for constitutive model evaluation in plasticity
}

\author{
A. Arsenlis *, N. R. Barton **, R. Becker, and R. E. Rudd \\ University of California \\ Lawrence Livermore National Laboratory \\ P.O. Box 808, Livermore, CA 94550
}

\begin{abstract}
A database storage, search and retrieval method of constitutive model responses for use in plasticity simulations is developed to increase the computational efficiency of finite element simulations employing complex non-linear material models. The method is based in the in situ adaptive tabulation method that has been successfully applied in the field of combustion chemistry, but is significantly modified to better handle the system of equations in plasticity. When using the database, the material response is estimated by a linear extrapolation from an appropriate database entry. This is shown to provide a response with an acceptable error tolerance. Two different example problems are chosen to demonstrate the behavior of the constitutive model estimation technique: a dynamic shock simulation, and a quasi-static inhomogeneous deformation simulation. This generalized in situ adaptive tabulation method shows promise for enabling simulations with complex multi-physics and multi-length scale constitutive descriptions.
\end{abstract}

Key words:

PACS:

\section{Introduction}

The complexity of continuum constitutive models used in finite element simulations has increased due to demands for better representation of material behavior and the availability of ever improving computational capabilities. Some of the most complex constitutive models are found in concurrent multiscale

* Fax: (925) 422-4665, Email Address: arsenlis@llnl.gov

**Email Address: barton22@llnl.gov 
modeling simulations in which the mechanical response at an integration point within the finite element simulation is determined by embedded simulations of another boundary value problem at a lower length scale. The embedded simulations may consist of smaller finite element models, dislocation dynamics, molecular dynamics, or ab initio simulations[1-4]. Somewhat less complex is the broad class of continuum internal state variable models that evolves a set of variables chosen to represent the evolving state of the material. The evolution equations for these variables often involve coupled nonlinear expressions that must be solved simultaneously with the thermo-mechanical response of the model. Both of the these methods may involve a significant number of degrees of freedom whose values do not directly enter into the global boundary value problem but are nonetheless necessary for its solution.

Despite the exponential increase in computing power, scientists and engineers must still be intelligent in solving time varying boundary value problems with finite element methods. Inefficient solutions methods can quickly overwhelm even the largest computers available, both in memory and CPU time. Perhaps the most widely adopted methods to raise the efficiency of finite element simulations are the methods of adaptive mesh refinement (AMR)[5]. AMR techniques search for optimal heterogeneous mesh point distributions within bodies to minimize the number of elements needed to solve particular boundary value problems to a desired degree of accuracy. During the course of simulations, the discretization is time dependent and may vary as a function of relative gradients in the problem solution.

While AMR techniques increase efficiency by optimizing the discretization of space, the complex material models are usually calculated independently at every integration point in a finite element mesh without regard for potential redundancy in these calculations. For highly complex constitutive models with many internal degrees of freedom, the calculation of the evolution of internal state variables and the response of the constitutive model consumes most of the CPU time during a simulation. Information gained from constitutive model evaluation in one part of the body is typically not communicated to other parts of the body or to other instants in time, with each integration point in the finite element geometry running its own instance of the constitutive model. For simple constitutive models, this approach may be the most efficient mode of operation due to the cost of tabulating and communicating the material response between integration points; however, for complex models, reuse of previously computed responses to specific loading conditions can significantly raise the efficiency of the overall simulation.

For example, consider a finite element simulation of homogeneous tensile elongation of a metallic single crystal. Take the constitutive model used at the integration points to be a dislocation dynamics simulation with periodic boundary conditions in which the internal state variables are the positions of the dis- 
location lines within the periodic cell. The communication between the finite element simulation and dislocation dynamics simulation involves the finite element system applying a stress to the dislocation dynamics simulation over a set time increment and the dislocation dynamics simulations updating the positions of all the dislocation lines returning the strain increment over that time period to the finite element simulation. The dislocation dynamics simulations providing the material response are orders of magnitude more computationally intensive than the finite element simulation that drives them. If replicas of the dislocation dynamics simulation cell are used to calculate the material response at all of the material points, the same simulation is run as many times as there are material points in the finite element simulation at each iteration. If, however, the information of material response at a single material point is communicated to all the others, the efficiency of the total simulation increases by a factor proportional to the number of integration points in the homogeneous finite element system.

There has been limited investigation of numerical methods that increase the efficiency of simulations by storing and communicating previously computed constitutive material responses between integration points in continuum simulations. One notable example is the in situ adaptive tabulation (ISAT) method developed in the area of combustion modeling [6,7]. In the ISAT method, a searchable database of constitutive model responses to known loading and internal variable states is built on the fly as the simulation proceeds. When a constitutive response at an integration point is needed, the system either returns a tabulated response from the database or runs the underlying complex constitutive model and adds the resulting response to the database. The database is built on the fly because the portion of the state variable space that is visited during solution of the boundary value problem is only a small subset of the total state variable space. The storage needed for a pre-computed database of all possible states would be immense, and even if it were possible to store that database, the efficiency of the search algorithms decreases with the increasing database size [8]. The ISAT method has been shown to be successful in a variety of boundary value problems in reactive chemistry that develop well defined process zones for extended periods of simulation time, such as those found in flame propagation problems [6].

In this article, we describe an in situ database construction and retrieval method applied to constitutive model evaluation and estimation for simulations of plastically deforming solids. To demonstrate the methodology, we employ a continuum crystal plasticity constitutive model because of its high degree of nonlinearity and large number of internal state variables. The constitutive model is described in Section 2. The construction, search and retrieval methods of the in situ database are described in Section 3. The method is applied to several example problems whose geometries and results are described in Section 4. We have chosen two time varying boundary value problems to 
test the generality and performance of the method: a dynamic shock simulation of a single crystal and the deformation behavior of a rectangular plate with a hole. We conclude with a short discussion of the implications of the results on directions for further research in the area of efficient material model evaluation methods.

\section{Continuum Crystal Plasticity Model Description}

The material constitutive model employed here describes the thermoelastic and viscoplastic response of crystals. The formulation accounts for finite strains and rotation of the crystal lattice with deformation. The inelastic deformation occurs by slip on specific crystallographic systems with the slip rate determined by the resolved shear stress on that system as well as its state of hardening.

The crystal plasticity model parallels existing models for finite-strain deformation of elasto-viscoplastic crystals $[9,10]$ with a notable modification to handle finite volume strains that occur in shock calculations. Particulars of the constitutive model are not the focus of the work presented here; some details are contained in the appendix.

In integrating the constitutive model, material state variables at the beginning of the time increment and the velocity gradient over the time increment are given. The system of equations outlined in the appendix is solved implicitly, and the stress and state variables at the end of the time step are computed.

\subsection{Incremental update of the material state}

In all of the examples presented here, a boundary value problem is solved using a finite element formulation with the material constitutive response given by the crystal plasticity model. In this context, the role of the constitutive model is to provide the thermo-mechanical response of the material at each finite element integration point. The material state must also be simultaneously updated and maintained for proper history dependence of the material response. If the finite element solution is using an implicit time integration scheme, the material model must also provide a material stiffness. For the update of the state descriptors for a single crystal, the inputs and outputs may be organized into vectors

$$
\mathbf{i}=\left\{\Delta t, \overline{\mathbf{L}}_{\mathrm{o}}(9), e, \overline{\mathbf{V}}^{\prime}(5), \ln (\operatorname{det}(\mathbf{V})), h\right\}
$$


and

$$
\mathbf{o}=\left\{\overline{\mathbf{V}}^{\prime}(5), \mathbf{q}_{\Delta}(4), h\right\} .
$$

The input vector consists of the time step $\Delta t$, the velocity gradient $\mathbf{L}_{\mathrm{o}}$, the internal energy $e$, the thermo-elastic strain components, $\mathbf{V}^{\prime}$ and $\ln (\operatorname{det}(\mathbf{V}))$, and the strength $h$. The number of unique scalar quantities composing the vector and tensor parts are given in parentheses. In order to reduce the input vector size by eliminating explicit dependence on the crystal orientation, the velocity gradient $\mathbf{L}$ and the deviatoric thermo-elastic strain $\mathbf{V}^{\prime}$ are rotated into a reference crystal lattice frame to obtain $\overline{\mathbf{L}}_{\mathrm{o}}$ and $\overline{\mathbf{V}}^{\prime}$.

The output vector consists of the updated deviatoric thermo-elastic strain $\overline{\mathbf{V}}^{\prime}$, a quaternion $\mathbf{q}_{\Delta}$ that parameterizes the incremental rotation of the crystal lattice over the time step $\Delta t$, and the updated strength variable $h$. If an implicit integration scheme is employed to solve the finite element system of equations, then the material model must also provide $\mathbf{J}=\partial \mathbf{o} / \partial \mathbf{i}$ to enable calculation of the material stiffness.

In terms of these input and output vectors, the task of the material model is then to compute

$$
\mathbf{o}=\mathcal{M}[\mathbf{i}],
$$

where $\mathcal{M}$ can be thought of as a complex nonlinear transfer function converting inputs $\mathbf{i}$ into ouputs o. Solving the highly nonlinear equations for the crystal response described by $\mathbf{o}$ is computationally expensive. In principle the response also includes the stress and the internal energy needed by the coarse model, but they are comparatively inexpensive to compute once $\mathbf{o}$ is known.

\section{Constitutive Model Approximation}

The goal of this work is to increase the efficiency of finite element simulations by reusing information obtained from evaluations of the constitutive model. This reuse reduces the frequency at which the set of coupled nonlinear equations in the constitutive model is solved.

By storing previously computed data sets of $\left.\mathbf{i}\right|_{\text {ref }},\left.\mathbf{o}\right|_{\text {ref }}$, and $\left.\mathbf{J}\right|_{\text {ref }}$ from evaluations of the nonlinear transfer function $\mathcal{M}$ in a searchable database; the constitutive model response may be estimated. For a given input, $\mathbf{i}$, an output $\left.\mathbf{o}\right|_{\text {est }}$ may be estimated from one of the previously computed data sets by

$$
\left.\mathbf{o}\right|_{\text {est }}=\left.\mathbf{o}\right|_{\text {ref }}+\left.\mathbf{J}\right|_{\text {ref }}\left(\mathbf{i}-\left.\mathbf{i}\right|_{\text {ref }}\right) .
$$


This equation corresponds to a truncated Taylor series expansion about the known value. In implicitly integrated finite element simulations, the thermomechanical jacobian must also be determined.

The first challenge of the approximation strategy is to limit the output error such that

$$
\left\|\mathbf{C}_{\mathrm{o}}\left(\mathcal{M}[\mathbf{i}]-\left.\mathbf{o}\right|_{\text {est }}\right)\right\| \leq \epsilon_{\mathrm{o}},
$$

where $\mathbf{C}_{\mathrm{o}}$ is a diagonal weighting matrix that normalizes and scales the relative importance of various components of the output vector, and $\epsilon_{\mathrm{o}}$ is a preset error tolerance. An error more difficult to manage is the one that may accumulate in the system from successive approximations of path dependent state variables rather than actual evaluations of the complex constitutive model. We do not formally address these possible cumulative errors, but through a few example applications, we show that such errors either are small or do not appear to propagate. The second challenge of the approximation strategy is to develop a cheap (in the computational sense) method to find the best previously computed data set stored in a database for use in the extrapolations. The rest of the section discusses our treatment of these two challenges.

\subsection{Ellipsoids of Accuracy}

The first challenge is to identify the region of the input space associated with each database entry $\left.\mathbf{i}\right|_{\text {ref }}$ in which the linear extrapolation estimate (Equation 4) lies within the error tolerance (Equation 5). This subject has received much attention in Pope's work [6]. He points out that a good estimate for the error in the linear approximation could be based on the second derivative of the output vector with respect to the input vector, but computation of that second derivative is often expensive. Its expense could easily make the approximation method more computationally intensive than calculating the response directly through the nonlinear transfer function. Rather than computing the second derivative to estimate the accuracy of the extrapolation, Pope employs a hyper-dimensional Ellipsoid of Accuracy (EOA) whose enclosed region contains points for which there is a desired level of confidence in the extrapolated value. The EOAs for the reference points evolve with the information gained from actual constitutive model evaluations. They expand to include new points that meet the error tolerance. The EOAs provide a very compact means of keeping track of the information provided by previous constitutive model calculations on the region of validity around each reference point. We borrow the concept of EOAs for our reference points from Pope, but we significantly modify the expansion algorithm to better handle problems in plasticity. 
An EOA of a previously computed data set in the output vector space may be defined by the set of vectors $\mathbf{o}$ that satisfy the following condition

$$
\left\|\mathbf{A}\left(\mathbf{o}-\left.\mathbf{o}\right|_{\text {ref }}\right)\right\| \leq 1
$$

where $\mathbf{A}$ is a matrix that describes the shape of the ellipsoid in the output vector space. Note that Equation 6 describes a solid hyper-ellipsoid in the output vector space.

Following Pope, we initialize A conservatively such that the EOA for a reference data point contains those points for which the error tolerance given by Equation 5 would be satisfied simply by the reference value $\left.\mathbf{o}\right|_{\text {ref }}$ if the response function $\mathcal{M}$ were strictly linear. Taking $\left.\mathbf{o}\right|_{\text {est }}=\left.\mathbf{o}\right|_{\text {ref }}$ and $\mathcal{M}=$ $\left.\mathbf{o}\right|_{\text {ref }}+\left.\mathbf{J}\right|_{\text {ref }}\left(\mathbf{i}-\left.\mathbf{i}\right|_{\text {ref }}\right)$ in Equation 5 we arrive at the initial condition $\mathbf{A}=\frac{1}{\epsilon_{\mathrm{o}}} \mathbf{C}_{\mathrm{o}}$. A will evolve according to the algorithm described below to account for information from subsequent constitutive model evaluations.

Using Equation 4 to estimate o, Equation 6 is equivalent to

$$
\left\|\left.\mathbf{A} \mathbf{J}\right|_{\text {ref }}\left(\mathbf{i}-\left.\mathbf{i}\right|_{\text {ref }}\right)\right\| \leq 1
$$

Because the input vector space has higher dimension than the output vector space, the region of accuracy is not closed in the input space. Input vector directions in the null space of $\left.\mathbf{J}\right|_{\text {ref }}$ do not contribute to the distance measure determining the EOA. The region of accuracy in the input space is closed by adding the condition

$$
d_{\mathrm{i}} \leq \epsilon_{\mathrm{i}} \quad, \quad d_{\mathrm{i}}=\left\|\mathbf{C}_{\mathrm{i}}\left(\mathbf{i}-\left.\mathbf{i}\right|_{\mathrm{ref}}\right)\right\| \quad,
$$

where $\mathbf{C}_{\mathrm{i}}$ is a diagonal matrix that normalizes and scales the components of input vectors, and $\epsilon_{\mathrm{i}}$ is an input error tolerance. In practice, $\epsilon_{\mathrm{i}}$ is chosen such that a given input will fail satisfy Equation 7 before it fails to satisfy Equation 8 unless it is closely aligned with a vector in the null space of $\left.\mathbf{J}\right|_{\text {ref }}$.

If $\mathcal{M}$ [i $]$ yields a point in the output vector space initially outside the EOA satisfying Equation 5, then the EOA may be grown to include that point. Whereas Pope employs a growth algorithm that finds the minimum volume concentric ellipsoid that completely contains the current ellipsoid and the new point, we diverge from his method and employ a somewhat more general algorithm that allows the center of the ellipsoid to change. Our EOA update algorithm takes the following form with the previously computed data set information transforming into the new primed quantities

$$
\mathbf{A}^{\prime}=\mathbf{B A}
$$




$$
\begin{aligned}
& \left.\mathbf{i}\right|_{\text {ref }} ^{\prime}=\left.\mathbf{i}\right|_{\text {ref }}+\frac{\eta_{*}-a}{\eta_{*}}\left(\mathbf{i}-\left.\mathbf{i}\right|_{\text {ref }}\right) \\
& \left.\mathbf{o}\right|_{\text {ref }} ^{\prime}=\left.\mathbf{o}\right|_{\text {ref }}+\left.\frac{\eta_{*}-a}{\eta_{*}} \mathbf{J}\right|_{\text {ref }}\left(\mathbf{i}-\left.\mathbf{i}\right|_{\text {ref }}\right) \\
& \left.\mathbf{J}\right|_{\text {ref }} ^{\prime}=\left.\mathbf{J}\right|_{\text {ref }}
\end{aligned}
$$

where

$$
\begin{aligned}
\mathbf{B} & =\frac{1}{b} \mathbf{I}+\left(\frac{1}{a}-\frac{1}{b}\right) \mathbf{n}_{*} \otimes \mathbf{n}_{*} \\
a & =\frac{(2+m) \eta_{*}+\sqrt{4+4 m+\eta_{*}^{2} m^{2}}}{4+2 m} \\
b & =\sqrt{\frac{1}{2} \beta-\sqrt{\beta^{2}-4 a^{2}}} \text { with } \beta=1+2 a \eta_{*}-\eta_{*}^{2} \\
\mathbf{n}_{*} & =\frac{\left.\mathbf{A} \mathbf{J}\right|_{\text {ref }}\left(\mathbf{i}-\left.\mathbf{i}\right|_{\text {ref }}\right)}{\left\|\left.\mathbf{A} \mathbf{J}\right|_{\text {ref }}\left(\mathbf{i}-\left.\mathbf{i}\right|_{\text {ref }}\right)\right\|} \\
\eta_{*} & =\left\|\left.\mathbf{A} \mathbf{J}\right|_{\text {ref }}\left(\mathbf{i}-\left.\mathbf{i}\right|_{\text {ref }}\right)\right\|
\end{aligned}
$$

and $m$ is equal to one less than the dimension of the output space for minimization of the EOA volume increase.

If $m$ is set to a value other than one less than the dimension of the output space, the objective function being minimized is different than the minimum volume ellipsoid. Other objective functions may be attractive because of the manner in which the EOA growth takes place. In the limit $m \rightarrow \infty$, the Pope solution is recovered for which the minimum volume ellipsoid is found with the additional constraint of a fixed center. For $m=0$, the ellipsoid does not extend in the $-\left(\mathbf{i}-\left.\mathbf{i}\right|_{\text {ref }}\right)$ direction further than the previous ellipsoid but may significantly expand in directions orthogonal to $\left(\mathbf{i}-\left.\mathbf{i}\right|_{\text {ref }}\right)$. Choices between these two extreme values of $m$ can be viewed as mixtures of the two extreme solutions. For all values of $m$ the entire region enclosed by the previous EOA is enclosed within the new EOA. Figure 1 shows three examples of different values of $m$ used to control the ellipsoid growth for the same initial EOA and new point in the output vector space to be included in a new EOA.

Since there is a strong hysteresis in the stress-strain behavior of a plastically deforming solid, the limit $m \rightarrow \infty$ solution employed by Pope could potentially cause significant error to enter the solution. If an EOA were grown by sampling points on the loading curve and allowed to extend symmetrically in the unloading direction, estimating the unloading behavior through the database entries could result in extrapolation errors that are orders of magnitude larger than $\epsilon_{\mathrm{o}}$. To solve this potentially serious problem, the simplest solution was to retain the structure of ellipsoid regions describing the limits of reliable extrapolation and move the ellipsoid centers. Alternatively, a more 
complex hyper-dimensional surface could be employed to enclose the added high-confidence region.

The basic issue here is that the regions of confidence, whether they are EOAs or something else, necessarily need to expand to include points that have not been calculated explicitly in the constitutive model. The ability to make such an expansion is based on the smoothness properties of the response. Naturally, it is desirable to use as much information from previous calculations as possible when expanding these regions because if they are expanded too far they will include points for which the linear approximation does not meet the error tolerance. Once included in an EOA, points inside are never again tested to ensure they meet the tolerance. It is assumed that they do. The technique can compensate for the possibility of invalid points in an EOA by putting a new reference point sufficiently close to the EOA surface. In the resulting region of overlap of two EOAs, the points are evaluated according to the closer reference point, and this overlap may effectively excise invalid portions of an EOA. Even so, the method used to expand the EOAs affects both the accuracy and the efficiency of the computational model. It is typically impractical to save all of the results from previous constitutive model evaluations, and the EOAs provide a compact distillation of the relevant information. We have therefore retained the basic structure of the EOA, but generalized its implementation to give better performance in plasticity calculations.

Whereas the center of the EOA in Pope's update scheme remained the position where $\mathcal{M}[\mathbf{i}]$ was evaluated, the center of our EOA changes and no longer corresponds to the point of constitutive model evaluation. In our method, the center of the initial EOA is still within the updated EOA. Due to linearity, the extrapolation calculated in Equation 4 remains with respect to the initial reference point that was exactly calculated by the constitutive model although its exact position within the EOA is lost after the first update.

\subsection{Extended growth of EOAs}

EOA growth occurs when the error in the extrapolated solution is compared to $\mathcal{M}[\mathbf{i}]$ for an input vector $\mathbf{i}$ outside the EOA and determined to be less than the specified acceptable error $\epsilon_{\mathrm{o}}$. The expressions discussed thus far grow the EOA such that $\mathbf{i}$ is on the surface of new EOA. If $\left\|\mathbf{C}_{\mathrm{o}}\left(\mathcal{M}[\mathbf{i}]-\left.\mathbf{o}\right|_{\text {est }}\right)\right\|<$ $\epsilon_{\mathrm{o}}$, the efficiency of the simulation may be increased by extending the EOA past the point i. Aggressively growing ellipsoids past probing input points is particularly useful in explicit finite element simulations, as this extended growth can prevent excessive $\mathcal{M}$ evaluations for input vectors that fluctuate about the current EOA surfaces. 
Let

$$
e_{\mathrm{rel}} \equiv \frac{\left\|\mathbf{C}_{\mathrm{o}}\left(\mathcal{M}[\mathbf{i}]-\left.\mathbf{o}\right|_{\mathrm{est}}\right)\right\|}{\epsilon_{\mathrm{o}}}
$$

be the relative extrapolation error. If we assume that all of the error is due to the $p^{\text {th }}$ term in a Taylor series expansion about the center of the EOA, the EOA may be extended past $\mathbf{i}$ to the point $\mathbf{i}^{\prime}=\left.\mathbf{i}\right|_{\text {ref }}+\left(e_{\text {rel }}\right)^{-1 / p}\left(\mathbf{i}-\left.\mathbf{i}\right|_{\text {ref }}\right)$ where $\epsilon_{\mathrm{o}}$ is reached. A more conservative approach that caps the growth for $e_{\mathrm{rel}}=0$ is to extend growth by a factor

$$
g=g^{\prime}\left(\frac{1}{1+\left(g^{\prime p}-1\right) e_{\mathrm{rel}}}\right)^{\frac{1}{p}}
$$

where the parameter $g^{\prime}$ is the maximum growth factor. The growth factor $g$ has the property that it is always less than both $g^{\prime}$ and the maximum factor allowed by the Taylor series analysis $\left(\left(e_{\mathrm{rel}}\right)^{-1 / p}\right)$. Figure 2 shows the functional behavior of $g$ versus $e_{\text {rel }}$ for several values of $p$. As $p$ increases and the error is assumed to be associated with higher order terms in the Taylor series expansion, the growth factor becomes an increasingly conservative function of the relative error. The growth factor modifies the solution proposed above for the EOA growth by modifying the expression for $\eta_{*}$ such that it now becomes

$$
\eta_{*}=g\left\|\left.\mathbf{A} \mathbf{J}\right|_{\text {ref }}\left(\mathbf{i}-\left.\mathbf{i}\right|_{\text {ref }}\right)\right\| \text {. }
$$

\subsection{Database Structure of Extrapolation Data Sets}

The database that contains the collection of the previously evaluated data sets and the EOAs described above is given the form of an unstructured tree that is grown on the fly as required by the finite element simulation. The tree is non-unique and history dependent such that the order in which database entries are added determines their position in the tree. Each point in the tree contains a data set for extrapolation; the EOA; and pointers to its parent, to its first child, and to its next sibling. The search algorithm proceeds as follows.

An input vector query $\mathbf{i}$ is made, and the tree search is started from its base, and the value of $d_{\mathrm{i}}$ (Equation 8), of the parent is compared to the values found in its children. The tree search proceeds until the parent node has a smaller value of $d_{\mathrm{i}}$ than any of its children. That parent node is now considered the "closest point" in the tree. Given that our tree is non-unique, there is no guarantee that the "closest point" found at the end of the search is the closest point as measured by $d_{\mathrm{i}}$ of the entire tree; it is however likely to be a good point from which to estimate the response of the transfer function $\mathcal{M}$. 
If $\mathbf{i}$ is within the EOA of the "closest point" from Equation 7 and Equation 8 is satisfied, $\left.\mathbf{o}\right|_{\text {est }}$ is calculated using Equation 4 and returned to the finite element simulation. If $\mathbf{i}$ is outside the EOA of the "closest point," $\mathbf{o}=\mathcal{M}[\mathbf{i}]$ and $\mathbf{J}[\mathbf{i}]$ are calculated. If the $e_{\text {rel }}$ is less than or equal to unity, the EOA of the closest point is grown to encompass $\mathbf{i}$ using the method outlined in the previous subsections. Otherwise if $e_{\text {rel }}$ is larger than unity, a child node is added to the "closest point" with the data obtained from the constitutive model evaluation and the initialization of the EOA already discussed. Note that when a constitutive model evaluation is performed only the tree node found to be the "closest point" is modified.

For added efficiency, when a response is returned to the finite element simulation, the location of the "closest point" within the tree structure is stored. At the next time step, the queries search the subtree branching from their "closest points" of the previous time step. If the result of the subtree search finds an entry whose EOA includes the query point, the response is extrapolated. If the query is outside the EOA of the "closest point" in the subtree, a search of the entire tree is conducted for the "closest point." If the query point again enters the subtree whose parent is the previous "closest point," the subtree search is not repeated but rather the query jumps to the "closest point" found in the initial subtree search, and the database is modified as described in the previous paragraph with an evaluation of $\mathcal{M}$.

Figure 3 summarizes the algorithm used in providing a response to a constitutive model evaluation request.

\section{Illustrative Examples of Method Application}

Two example problems are chosen to illustrate the behavior of the method in different types of loading histories: a dynamic flyer-target impact, and quasistatic loading of a plate with a stress concentration. The method is well suited to the impact problem, in which the deformation is initially concentrated in compressive shocks, and these shocks approach steady state before they reach the free surface of the target. In the quasi-static problem, the monotonically increasing load results in continuous exploration of new regions of material state in the constitutive model. The quasi-static problem also involves substantial inhomogeneity due to the effects of the stress concentration. Despite the range of deformation conditions represented in the example problems, the method performs well in both cases.

Material properties appropriate to unalloyed copper are used for both example problems. Parameters are given in the appendix. Input and output vector weights, $\mathbf{C}_{\mathrm{i}}$ and $\mathbf{C}_{\mathrm{o}}$, are largely kept constant. The weights for $\Delta t$ and $\overline{\mathbf{L}}_{\mathrm{o}}$ 
are however adjusted according to the physics of each example problem. In general, it is important to adjust the weights so that the distance measures in the input and output vector spaces are not dominated or perverted by particular entries in an undesirable fashion.

To illustrate the behavior of the method, simulations are performed with EOA growth $m$ values of $m=0, m=9$, and $m \rightarrow \infty$. The $m=9$ case corresponds to ellipsoid growth with minimum volume increase in the actual dimension of the output vector space. The $m=0$ and $m \rightarrow \infty$ cases demonstrate the extremes of the ellipsoid growth solution.

\subsection{Dynamic Shock Loading}

This example problem was chosen to illustrate the performance of the method for situations with a fairly steady deformation process zone and to demonstrate application in an explicit finite element simulation. The experiments simulated are flyer-plate impact tests. The modeled configuration comprises a copper flyer disk $1 \mathrm{~cm}$ thick and traveling $200 \mathrm{~m} / \mathrm{s}$ impacting a $2 \mathrm{~cm}$ thick stationary single crystal copper target with a [100] orientation. The other dimensions of the flyer and target are large compared to the thickness so that the deformation is one dimensional uniaxial strain. The diagnostics typically associated with these experiments are velocity measurements made on the free surface. Gas guns, laser interferometry, and other technical details related to dynamic loading experiments are described in [11].

When the plates collide, elastic and plastic waves are generated at the impact surfaces of both flyer and target. At the current impact velocity, the elastic waves travel faster than the plastic waves, creating a noticeable step in the through thickness stress profile. When a wave strikes the free surface, it is reflected and the free surface accelerates. The reflected wave relieves the stress. The time evolution of the surface velocity indicates the time history of the stress wave impacting the surface, so that features like the elastic-plastic transition are evident as shown in the rising velocity in Figure 4. Velocity at the free surface is zero until the arrival of the first wave after 4 microseconds. The early time results where the velocity is zero are omitted. Following the wave reflection, the free surface velocity is constant until another wave strikes the surface.

The 2:1 thickness ratio of target to flyer results in the stress wave in the flyer being reflected and reaching the target impact face at roughly the same time as the original wave in the target reaches the target free surface. As the relief wave originating from the flyer strikes the free surface, it drops the surface velocity to nearly zero, as seen in Figure 4. Features evident on this portion 
of the velocity history are the reflected elastic-plastic transition from the flyer and additional features associated with yield on stress reversal.

All of the simulations in this section are performed with an error tolerance of $\epsilon_{\mathrm{o}}=10^{-3}$. The adaptive tabulation method is used only in the target in order to simplify interpretation of the data. All simulations require roughly fifteen thousand time steps to reach completion.

In Figure 4, the reference free surface velocity profile is obtained from a simulation without adaptive tabulation (all constitutive model queries are satisfied by performing a full nonlinear constitutive model evaluation). Figure 4 also reports the magnitudes of the free surface velocity error for the three $m$ cases, all with no extended EOA growth (with $g^{\prime}=1$ in Equation 19). Note, the velocity errors (right axis) are plotted on a different scale than the velocity itself (left axis). The free surface velocity is part of the overall simulation response and is not a direct output of the constitutive model. It is therefore not directly controlled by the preset error tolerances. Given the value of $\epsilon_{\mathrm{o}}$, velocity errors are well controlled on average, with the $m \rightarrow \infty$ case showing the largest peak errors.

For the same three cases, Figure 5 gives the evaluation fraction, the fraction of constitutive model queries that result in nonlinear constitutive model evaluations. Before the relief waves enter the target at approximately $4 \mu \mathrm{s}$, the evaluation fraction remains relatively low. In the $m=9$ and $m \rightarrow \infty$ cases, the evaluation fraction tends to be diminishing over much of this simulation time. This decrease happens as the elastic and plastic waves become distinct and approach steady profiles as they travel. As rarefaction waves tend to spread as they travel, the entrance of the relief waves into the target disrupts the trend to steady state and increases the evaluation fraction.

As we will see below, the evaluation fraction performance is improved dramatically through the use of extended EOA growth. Due to the physics and numerics involved, explicit simulations such as this one involve many waves of relatively small amplitude. This noise results in many constitutive model queries fluctuating about the boundary of the current EOA with the queries outside the EOA requiring full non-linear constitutive model evaluation. By extending the growth of an ellipsoid past a given query point, many of these future queries resulting from minor fluctuations can be eliminated.

Figure 6 contains the evaluation fraction, database size, and free surface velocity error for a suite of simulations in which the extended growth parameters, $g^{\prime}$ and $p$, are systematically varied. Results for $g^{\prime}=1$ are not plotted, as they correspond (for all $p$ ) to the cases given in Figure 5. For all three $m$ cases, extended growth gives a considerable reduction in evaluation fraction (top row of Figure 6). If the simulations were dominated by the time to evaluate 
the constitutive model, nearly two orders of magnitude in speedup would be observed. For a given set of extended growth parameters, the evaluation fraction for the $m=0$ case remains relatively high. In all $m$ values examined, as $p$ increases the extended growth is severely restricted and similar evaluation fractions are obtained regardless of $g^{\prime}$.

Some fraction of the constitutive model evaluations result in the creation of new database entries. The total database sizes at the end of the simulations are shown in the second row of Figure 6. More aggressive growth reduces the number of evaluations used to grow the EOAs, but it has a relatively small impact on the number of database entries needed to fill the required region of the output space. Therefore the database sizes do not vary as widely as the evaluation fractions.

Time-averaged velocity errors given in the bottom row of figure Figure 6 are obtained by averaging the magnitude of the velocity error in time, beginning at $4 \mu \mathrm{s}$. Velocities in all simulations are zero before $4 \mu \mathrm{s}$, and including this time range would artificially decrease the error measure. In general the errors are well controlled at this value of $\epsilon_{\mathrm{o}}$ for the range of extended growth parameters. Errors from the $m \rightarrow \infty$ case span a broader range and are larger than those observed in the other two cases.

Overall, the $m=0$ and $m=9$ cases give better error control for this example problem. The most conservative case here is $m=0$ because the loading is such that while load reversals are common the material points follow similar paths in the state variable space displaced in time. For larger $\epsilon_{\mathrm{o}}$ and certain ranges of the constitutive model parameters, the $m \rightarrow \infty$ case can result in unphysical output vectors. This pathology is due to the stiff nature of the ordinary differential equations in the constitutive model and the property of the $m \rightarrow \infty$ growth to extend the EOA too far in the unloading paths of the materials such that the error tolerances are violated. On the other hand, the $m=9$ and $m \rightarrow \infty$ cases yield greater computational efficiencies.

\subsection{Deformation of a Plate with a Hole}

In this example problem, a plate with a stress concentration is subjected to monotonic quasi-static loading. The plate is extended in the horizontal direction and allowed to contract in the vertical direction. That is, the top surface is traction free. Surfaces normal to the plane are also traction free. The cube directions of the crystal lattice are initially aligned with the sample directions so that, by symmetry, only one eighth of the plate needs to be simulated (shown in Figure 7). A cylindrical hole through the plate acts as a stress concentrator, and in Figure 7 we see that the strain localizes in a band 
near the hole. The plate is extended until the bulk of the domain is deforming plastically. Both the highest and the lowest effective strains are located at the edge of the hole.

The hole not only concentrates the stress but also results in changes to the deformation mode. The crystal lattice rotations and the non-proportional deformation make the problem more challenging for the adaptive tabulation method because it may use a larger region of a given EOA. Furthermore, the progressive overall loading means that the constitutive model is exploring new regions of its state space with each new time step. Adaptive tabulation method is nevertheless able to appreciably reduce the number of full non-linear constitutive model evaluations. In this problem, the reduction is largely due to database building during iterations within a given time step. An implicit time stepping scheme is used at the finite element level, and several iterations are required to obtain the velocity field solution for each time step. The evaluation fraction for the first iteration may be high, but as the finite element solution converges the changes to the velocity field solution decrease in magnitude and the evaluation fraction falls off rapidly with each new iteration.

The evaluation fraction and relative errors are explored as a function of the preset error tolerance $\epsilon_{\mathrm{o}}$ of the EOAs. The relative error is calculated for two quantities: the applied load, and the strain rate at a material location near the stress concentration. The absolute error is measured as a function of time with respect to a simulation in which all constitutive model queries are provided by full non-linear model evaluations. The relative errors are then calculated as the time averaged magnitude of these absolute errors divided by a constant representative value of the quantity in question. These error measures quantify only the modeling error in the adaptive tabulation method; as the evaluation fraction approaches unity the error goes to zero. Figure 8 shows the variation in these relative errors with $\epsilon_{\mathrm{o}}$.

The applied load is a quantity obtained by summing over all of the tractions on the loading face, and it is not surprising that this aggregate quantity has smaller errors for a given value of $\epsilon_{\mathrm{o}}$ than the error in local quantities like strain rate. The relative error in local strain rate may also be greater because the material point at which it is measured is in the plastic regime for a longer period of time than rest of the geometry and errors are largely absent in the elastic regime. For both error measures, $m=9$ tends to give the best results. In this example problem, unlike the previous example, $m=0$ does not appear to be the conservative choice. The fact that the slopes of the relative error curves tend to 1 as $\epsilon_{\mathrm{o}}$ becomes small is an indication that errors are well controlled.

The evaluation fractions associated with different preset error tolerances are shown in Figure 9. As mentioned above, much of the reduction in the full 
non-linear model evaluation is due to building the database during iterations for the velocity field solution of a given time step. As $\epsilon_{\mathrm{o}}$ approaches zero, more nonlinear constitutive model evaluations are required and the evaluation fraction approaches unity. Viewing figures 8 and 9 together, it is concluded that small evaluation fractions are achieved without inducing large errors.

By reflecting on the results of both examples, it appears that the $m=9$ case would be the best overall choice for the EOA growth scheme. This value of $m$ corresponded to minimizing the volume in the output space of our state vectors. In the material shock example, it provided acceptable responses as gauged by the preset error tolerance while requiring fewer full non-linear model evaluations than the $m=0$ case. In the example with the stress concentration, it provided the best responses while requiring fewer full non-linear model evaluations than the $m \rightarrow \infty$ case. It appears that if one is to apply these methods with little understanding of the trajectory of the system in the state space of the material model, setting the $m$ to one less than the output space dimension of the system is the best choice both in terms of accuracy and efficiency of the calculation.

\section{Conclusions}

In this article, we have demonstrated that the in situ adaptive tabulation (ISAT) method first developed for use in combustion simulations can also be applied to simulations of plasticity. Due to differences between the thermomechanical constitutive equations used in plasticity and chemical kinetics constitutive equations used in combustion simulations, the original ISAT method had to be modified. Whereas the extrapolation centers in the original ISAT database remain fixed, here the centers move as dictated by our modified growth algorithms. We find that our Ellipsoid of Accuracy growth scheme led to more accurate results, and that extending their growth when the errors were less than a present tolerance reduced the number of full non-linear constitutive model evaluations.

We have demonstrated the principle that building a searchable database of computed constitutive model evaluations and estimating material response through tabulations can significantly increase the efficiency of a finite element simulation if the computational expense is dominated by constitutive model evaluations. The base material model that we chose to illustrate the method was a rather simple crystal plasticity model when compared to other crystal plasticity models in the literature, so the actual time to run a simulations only increased by a small (but significant) factor. In the cases where the evaluation time for the constitutive model is a primary consideration, simulations that were computational unfeasible now may become possible with this paradigm 
used for constitutive model evaluation.

A current limitation of this method is that the database is not unique and history dependent, and there is no guarantee that the point in the database that is used to tabulate the constitutive response is the point that would yield the smallest error when compared to a full non-linear model evaluation. Other database structures whose search results in the best point from which to extrapolate the response to a given query need to be explored. Furthermore, distributed database storage techniques must also be investigated. In large problems with complex multi-variate constitutive models, database storage requirements quickly overwhelm the physical memory of individual workstations. The promise of this method to increase the efficiency of finite element simulations in concurrence with popular adaptive mesh refinement methods must also investigated.

\section{Appendix}

\section{A Material model details}

This section is meant only to convey the overall character of the constitutive model. More details may be found in [12] and [13] for similar crystal plasticity models.

Deformation of the crystallite is represented in terms of a multiplicative decomposition of the deformation gradient of the form [10]

$$
\mathbf{F}=\mathbf{V} \cdot \mathbf{R} \cdot \mathbf{F}^{\mathrm{p}}
$$

where $\mathbf{V}$ is a symmetric tensor that embodies both elastic and thermal lattice straining, $\mathbf{R}$ is the lattice rotation, and $\mathbf{F}^{\mathrm{P}}$ is deformation due to dislocation glide.

The velocity gradient $\mathbf{L}$ is then given by

$$
\begin{aligned}
& \mathbf{L}=\dot{\mathbf{F}} \cdot \mathbf{F}^{-1}=\dot{\mathbf{V}} \cdot \mathbf{V}^{-1}+\mathbf{V} \cdot \hat{\mathbf{L}} \cdot \mathbf{V}^{-1} \\
& \hat{\mathbf{L}}=\dot{\mathbf{R}} \cdot \mathbf{R}^{\mathrm{T}}+\mathbf{R} \cdot \overline{\mathbf{L}} \cdot \mathbf{R}^{\mathrm{T}} \quad, \quad \overline{\mathbf{L}}=\sum_{\alpha=1}^{n} \dot{\gamma}^{\alpha}\left(\overline{\mathbf{s}}^{\alpha} \otimes \overline{\mathbf{m}}^{\alpha}\right)
\end{aligned}
$$

Equation A.3 represents the slip rate $\dot{\gamma}^{\alpha}$ on a crystallographic slip system, $\alpha$, that is characterized by slip plane $\overline{\mathbf{m}}^{\alpha}$ and slip direction $\overline{\mathbf{s}}^{\alpha}$. The slip rate 
is a nonlinear function of the Cauchy stress projected on the slip system, as described below.

The Cauchy stress $\boldsymbol{\sigma}$ is determined by the relations

$$
\boldsymbol{\sigma}=\frac{1}{\operatorname{det}(\mathbf{V})} \hat{\boldsymbol{\tau}} \quad, \quad \hat{\boldsymbol{\tau}}=f_{\mathcal{K}}(\hat{\mathbf{E}}, e)
$$

governing the elastic response, where $e$ is the internal energy. The internal energy enters through the equation of state, such as the Mie-Grüneisen equation of state used here [14]. The logarithmic lattice strain measure $\hat{\mathbf{E}}=\ln (\mathbf{V})$ is used to facilitate decoupling of the volumetric and deviatoric elastic deformation.

For metals of cubic crystal symmetry, it is typically acceptable to assume that the distortional lattice strains are small even when the volume strains are large. This assumption permits substantial simplification of the kinematics. Thus, the volume strain is given exactly by $\operatorname{tr}(\hat{\mathbf{E}})=\ln (\operatorname{det}(\mathbf{V}))$, and the deviatoric lattice strain measure is approximated by $\hat{\mathbf{E}}^{\prime} \approx \mathbf{V}^{\prime}(\operatorname{det}(\mathbf{V}))^{-1 / 3}$, where a prime denotes the deviatoric part. The formulation is simplified appreciably by this assumption of small deviatoric lattice strains and by using components of the lattice strain and its time rate of change computed in a rotating frame.

The Kirchoff stress $\hat{\tau}$, expressed in terms of these strain variables, is projected onto the glide system to compute the driving force for slip. Under the simplifying lattice strain assumptions,

$$
\tau^{\alpha}=\hat{\boldsymbol{\tau}}:\left(\hat{\mathbf{s}}^{\alpha} \otimes \hat{\mathbf{m}}^{\alpha}\right)
$$

This resolved shear stress and the strength of the glide system determine the slip rate. In this simplified crystal constitutive model, a single variable $h$ is used to describe the strength of the crystallite. The slip rate is then computed according to a mechanical threshold stress based kinetics model [15] combined with drag-limited dislocation velocities $[11,16,17]$ :

$$
\begin{aligned}
& \dot{\gamma}^{\alpha}=\left(\frac{1}{\dot{\gamma}_{\mathrm{w}}^{\alpha}}+\frac{1}{\dot{\gamma}_{\mathrm{r}}^{\alpha}}\right)^{-1} \operatorname{sgn}\left(\tau^{\alpha}\right) \\
& \dot{\gamma}_{\mathrm{r}}^{\alpha}=\dot{\gamma}_{\mathrm{ro}}\left(1-\exp \left(-\frac{\left|\tau^{\alpha}\right|}{D_{\mathrm{r}}}\right)\right) \\
& \dot{\gamma}_{\mathrm{w}}^{\alpha}=\dot{\gamma}_{\mathrm{wo}} \exp \left(-\frac{\Delta G^{\alpha}}{k \theta}\right)
\end{aligned}
$$


with

$$
\Delta G^{\alpha}=\left\{\begin{array}{cc}
\mu^{\alpha} g_{\mathrm{o}} b^{3}\left(1-\left(\frac{\left|\tau^{\alpha}\right|-\tau_{\mathrm{a}}}{g^{\alpha}}\right)^{p}\right)^{* q} & \text { for }\left|\tau^{\alpha}\right|>\tau_{\mathrm{a}} \\
\mu^{\alpha} g_{\mathrm{o}} b^{3} & \text { for }\left|\tau^{\alpha}\right| \leq \tau_{\mathrm{a}}
\end{array}\right.
$$

and $g^{\alpha}=s \sqrt{h}$. The value of $\frac{\mu^{\alpha} g_{0} b^{3}}{k \theta}$ is typically large enough that for $\left|\tau^{\alpha}\right| \leq \tau_{\mathrm{a}}$ the value of $\dot{\gamma}_{\mathrm{w}}^{\alpha}$ is effectively zero. We have defined $x^{* q}=|x|^{q} \operatorname{sgn}(x)$. The drag stress $D_{\mathrm{r}}$ depends linearly on temperature. In the context of the work here, the dislocation glide is not drag-limited, but the drag effects are a physically meaningful way to control the numerics when inputs to the model may be prone to errors.

The evolution of the (dimensionless) material strength $h$ is calculated according to

$$
\dot{h}=\left(k_{1} \sqrt{h}-k_{2}(\dot{\gamma}) h\right) \dot{\gamma} \quad, \quad k_{2}(\dot{\gamma})=k_{2 \mathrm{o}}\left(\frac{\dot{\gamma}}{\dot{\gamma}_{\mathrm{s}_{\mathrm{o}}}}\right)^{-1 / n}
$$

where $\dot{\gamma}=\sum_{\alpha}\left|\dot{\gamma}^{\alpha}\right|$.

In this formulation, the complete state of a crystal is described by the following: the lattice strain measures $\mathbf{V}^{\prime}$ and $\ln (\operatorname{det}(\mathbf{V}))$; the lattice orientation, which is parameterized using quaternions $\mathbf{q}[18]$; and the variable describing the current material strength $h$.

Material parameters are given in Table A.1 and are chosen to be appropriate for unalloyed copper. Elasticity parameters are drawn from [19]. Parameters for the Mie-Grüneisen equation of state are drawn from [20] and are not repeated here. Parameters for thermally activated slip kinetics are from [15].

\section{References}

[1] F. Feyel, A multilevel finite element method $\left(\mathrm{fe}^{2}\right)$ to describe the response of highly non-linear structures using generalized continua, Compt. Methods Appl. Mech. \& Eng. 192 (28-30) (2003) 3233-3244.

[2] H. M. Zbib, T. D. de la Rubia, A multiscale model of plasticity, Int. J. Plasticity 18 (9) (2002) 1133-1163.

[3] R. E. Miller, E. B. Tadmor, The quasicontinuum method: Overview, applications and current directions.

[4] R. E. Rudd, J. Q. Broughton, Coupling of length scales in solid state systems, Physica Status Solidi (b) 217 (251-291). 
Table A.1

\begin{tabular}{llllll}
\hline \hline$k_{1}$ & $k_{2 \mathrm{o}}$ & $n$ & $\dot{\gamma}_{\text {wo }}$ & $s$ & $h_{t=0}$ \\
\hline 98 & 7.7 & 100 & $10^{3} \mu \mathrm{s}^{-1}$ & $25 \mathrm{MPa}$ & 36
\end{tabular}

\begin{tabular}{lllll}
\hline \hline$c_{11}$ & $c_{12}$ & $c_{44}$ & $c_{\mathrm{v}}$ & $\bar{\rho}$ \\
\hline $176.374 \mathrm{GPa}$ & $129.204 \mathrm{GPa}$ & $75.188 \mathrm{GPa}$ & $0.385 \frac{\mathrm{kJ}}{\mathrm{kg} \cdot \mathrm{K}}$ & $8.93 \frac{\mathrm{g}}{\mathrm{cm}^{3}}$ \\
\hline \hline
\end{tabular}

\begin{tabular}{ccccc}
$\left(g_{\mathrm{o}} b^{3}\right) / k$ & $p$ & $q$ & $\tau_{\mathrm{a}}$ & \multicolumn{1}{l}{$\dot{\gamma}_{\text {wo }}$} \\
\hline $1.944 \mathrm{~K} / \mathrm{MPa}$ & $2 / 3$ & 1 & $40 \mathrm{MPa}$ & $8.08 \cdot 10^{3}$ \\
\hline \hline$\dot{\gamma}_{\mathrm{ro}}$ & $D_{\mathrm{r}}$ & $\frac{\partial D_{\mathrm{r}}}{\partial \theta}$ & \\
\hline $10^{5} \mu \mathrm{s}^{-1}$ & $386.5 \mathrm{MPa}$ & $1.29 \mathrm{MPa} / \mathrm{K}$ \\
\hline \hline
\end{tabular}

Material parameters

[5] T. Plewa, T. Linde, V. G. Weirs (Eds.), Adaptive Mesh Refinement - Theory and Applications, Springer, 2005.

[6] S. B. Pope, Computationally efficient implementation of combustion chemistry using in situ adaptive tabulation, Combust. Theory Modelling 1 (1997) 41-63.

[7] M. A. Singer, S. B. Pope, Exploiting ISAT to solve the reaction-diffusion equation, Combust. Theory Modelling 8 (2) (2004) 361-383.

[8] I. Veljkovic, P. E. Plassmann, D. C. Haworth, A scientific on-line database for efficient function approximation, in: V. Kumar, M. L. Gavrilova, C. J. K. Tan, P. L'Ecuyer (Eds.), ICCSA (1), Vol. 2667 of Lecture Notes in Computer Science, Springer, 2003, pp. 643-653.

[9] P. E. McHugh, R. J. Asaro, C. F. Shih, Crystal plasticity models, in: S. Suresh, A. Mortensen, A. Needleman (Eds.), Fundamentals of Metal-Matrix Composites, Butterworth-Heinemann, 1993, Ch. 8.

[10] E. B. Marin, P. R. Dawson, On modelling the elasto-viscoplastic response of metals using polycrystal plasticity, Compt. Methods Appl. Mech. \& Eng. $165(1-4)(1998) 1-21$.

[11] M. A. Meyers, Dynamic Behavior of Materials, John Wiley \& Sons, Inc., 1994.

[12] N. R. Barton, D. J. Benson, R. Becker, Crystal level continuum modeling of phase transformations: the $\alpha \leftrightarrow \epsilon$ transformation in iron, submitted to Modelling and Simulation in Materials Science and Engineering... accepted by reviewers and hopefully in print by the time this article needs to be in final form (2005).

[13] N. R. Barton, D. J. Benson, R. C. Becker, Crystal level simulations using Eulerian finite element methods, in: Materials processing and design: modeling, simulation and applications, Vol. 712 of AIP Conference Proceedings, NUMIFORM, Columbus, Ohio, 2004, pp. 1624-1629. 
[14] D. S. Drumheller, Introduction to wave propagation in nonlinear fluids and solids, Cambridge University Press, 1998.

[15] P. S. Follansbee, U. F. Kocks, A constitutive description of the deformation of copper based on the use of the mechanical threshold stress as an internal state variable, Acta Metall. 36 (1) (1988) 81-93.

[16] P. S. Follansbee, High strain rate deformation in fcc metals and alloys, Tech. Rep. LA-UR-85-3026, Los Alamos National Laboratory (1985).

[17] M. Hiratani, E. M. Nadgorny, Combined model of dislocation motion with thermally activated and drag-dependent stages, Acta Mater. 49 (20) (2001) 4337-4346.

[18] S. L. Altmann, Rotations, Quaternions, and Double Groups, Oxford University Press, 1986.

[19] A. Kelly, G. W. Groves, P. Kidd, Crystallography and crystal defects, Revised Edition, Wiley, 2000.

[20] D. J. Steinberg, Equation of state and strength properties of selected materials, Tech. Rep. UCRL-MA-106439, Lawrence Livermore National Laboratory (1996).

This work was performed under the auspices of the U.S. Department of Energy by University of California, Lawrence Livermore National Laboratory under contract W-7405-Eng-48. 
8
$\uparrow$
ह

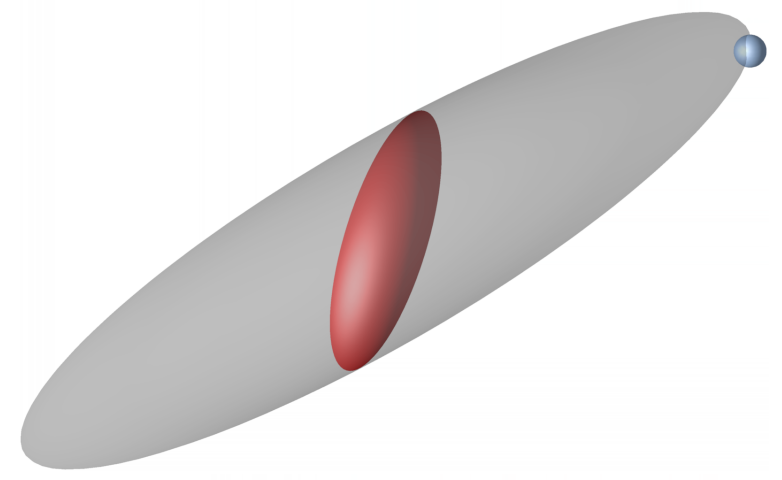

$N$
$\|$
$\varepsilon$

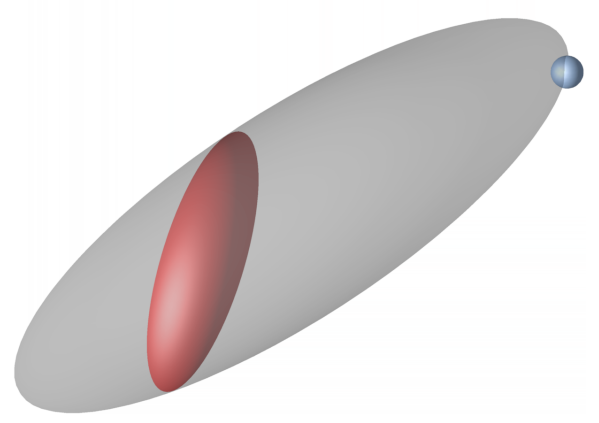

0
$\|$
E

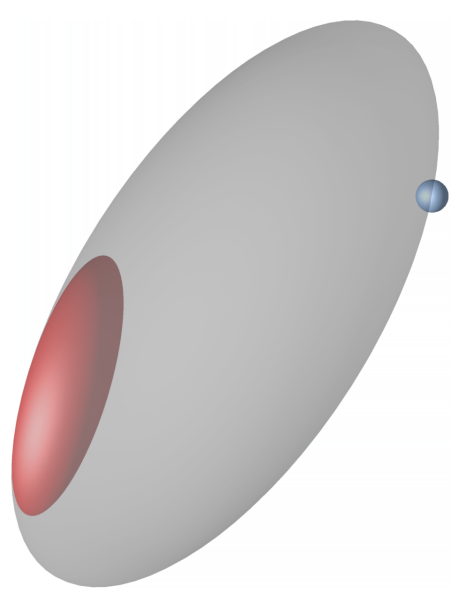

Fig. 1. Ellipsoid growth examples 


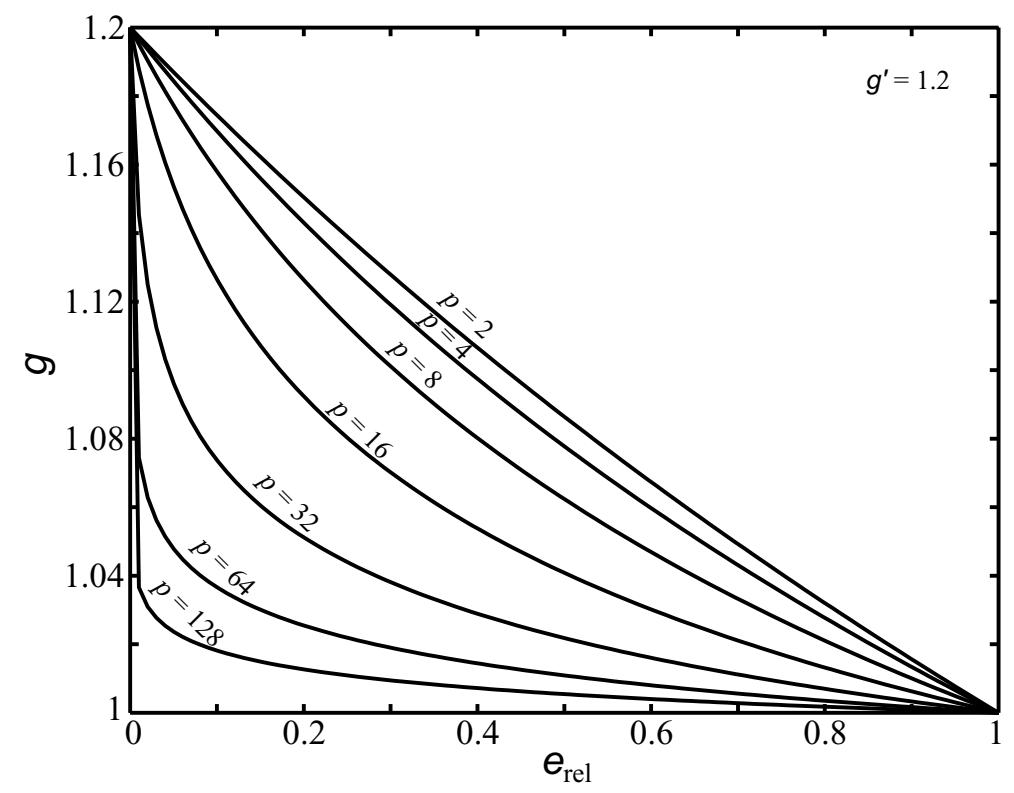

Fig. 2. Extended growth factor as a function of the error relative to the error tolerance with a maximum growth factor set to 1.2 
- Retrieve the stored constitutive model state for the beginning of the time step

- Retrieve the tree point that was used in answering the previous constitutive query for this finite element point; use it as the guess in starting the tree search

- Form the input vector $\mathbf{i}$ for the current query

- Find the "closest point" in the tree: if $\left\|\left.\mathbf{A} \mathbf{J}\right|_{\text {ref }}\left(\mathbf{i}-\left.\mathbf{i}\right|_{\text {ref }}\right)\right\| \leq 1$

(Equation 7) is satisfied for the guess tree point then \{

- Return the guess as "closest point"

\} else \{

- Search the sub-tree starting at the guess

- if $\left(\left\|\left.\mathbf{A} \mathbf{J}\right|_{\text {ref }}\left(\mathbf{i}-\left.\mathbf{i}\right|_{\text {ref }}\right)\right\| \leq 1\right)$ for the closest point found in the sub-tree then return it as "closest point" $\}$ else \{Start the search from the top of the tree to obtain "closest point"

\}

- Retrieve $\left.\mathbf{i}\right|_{\text {ref }},\left.\mathbf{o}\right|_{\text {ref }}$, and $\left.\mathbf{J}\right|_{\text {ref }}$ for the "closest point"

- Compute $\left.\mathbf{o}\right|_{\text {est }}=\left.\mathbf{o}\right|_{\text {ref }}+\left.\mathbf{J}\right|_{\text {ref }}\left(\mathbf{i}-\left.\mathbf{i}\right|_{\text {ref }}\right)$

- if for "closest point" $\left(\left\|\left.\mathbf{A} \mathbf{J}\right|_{\text {ref }}\left(\mathbf{i}-\left.\mathbf{i}\right|_{\text {ref }}\right)\right\| \leq 1\right)$ and Equation 8 is satisfied then \{

- Use o $\left.\right|_{\text {est }}$ for the output

\} else \{

- Run the constitutive model to determine $\mathbf{o}=\mathcal{M}[\mathbf{i}]$ and $\mathbf{J}[\mathbf{i}]$

- if the condition in Equation 8 is violated then \{

- Add a tree point at $\mathbf{i}$

\} else \{

- Compute the error $e_{\mathrm{o}}=\left\|\mathbf{C}_{\mathrm{o}}\left(\mathbf{o}-\left.\mathbf{o}\right|_{\text {est }}\right)\right\|$

- if $e_{\mathrm{o}} \leq \epsilon_{\mathrm{o}}$ (see Equation 5) then \{

- Compute $\eta_{*}$ using Equation 20

- Compute $\mathbf{B}$ using $\eta_{*}$ and Equations 13 through 16

- Update the tree point using Equations 9 through 12

\} else Add a tree point at $\mathbf{i}$ \}

\}

\}

- Store the updated constitutive model state; compute stress and material tangent stiffness as needed for the finite element code

Fig. 3. Outline of constitutive query algorithm. 


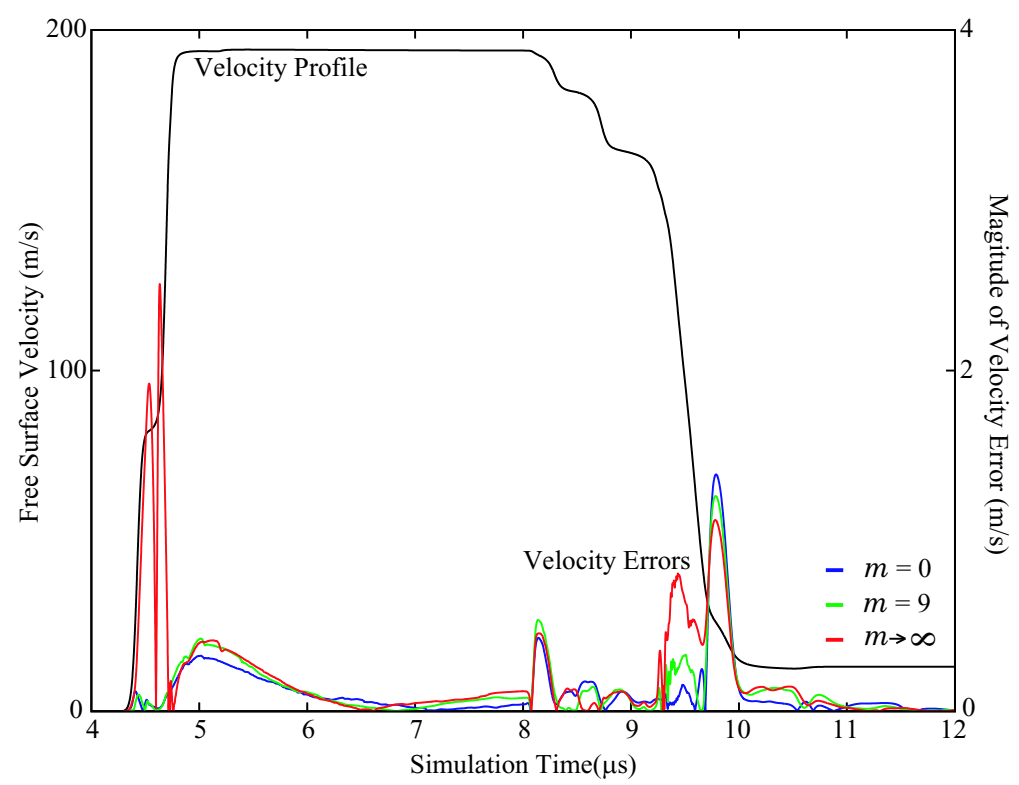

Fig. 4. Impact simulation results.

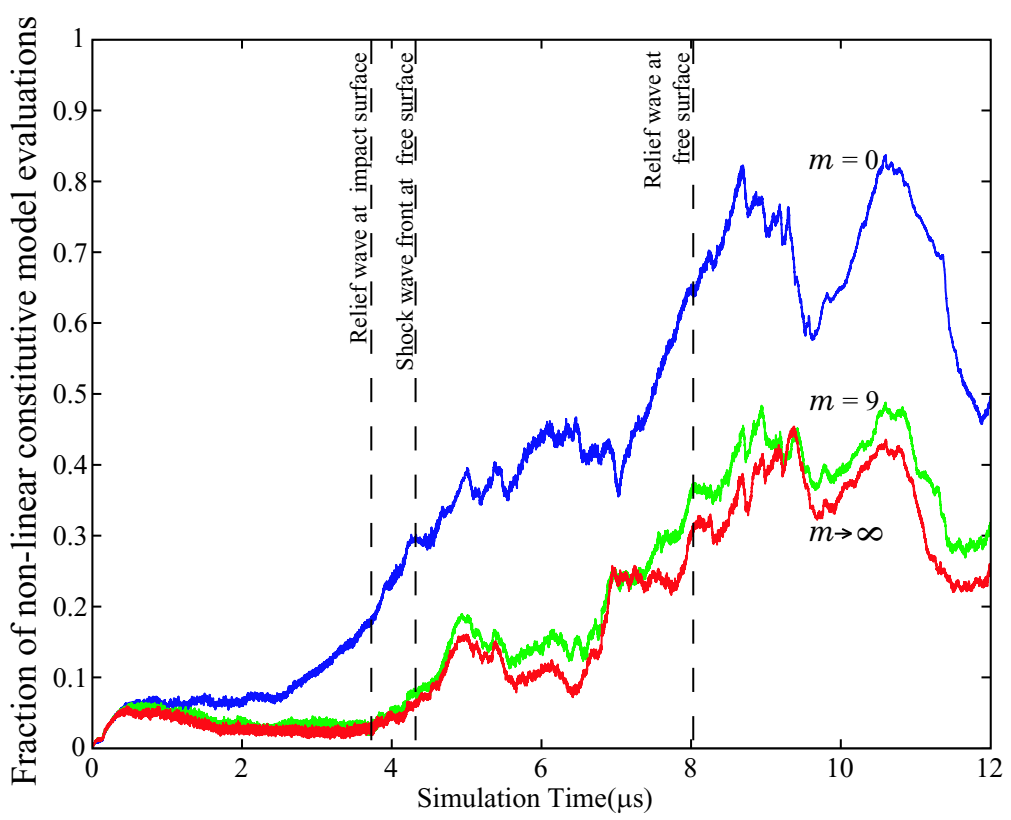

Fig. 5. Impact simulation results. The fraction of non-linear constitutive model evaluations is $0.39,0.20$, and 0.17 for the $m=0, m=9$, and $m \rightarrow \infty$ cases respectively. 

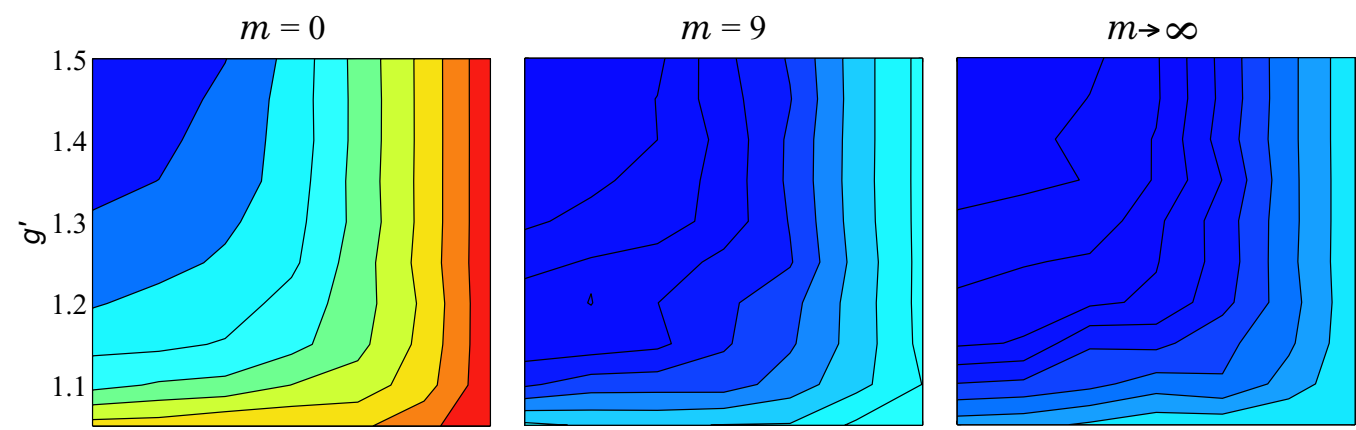

Fraction of non-linear constitutive model evaluations

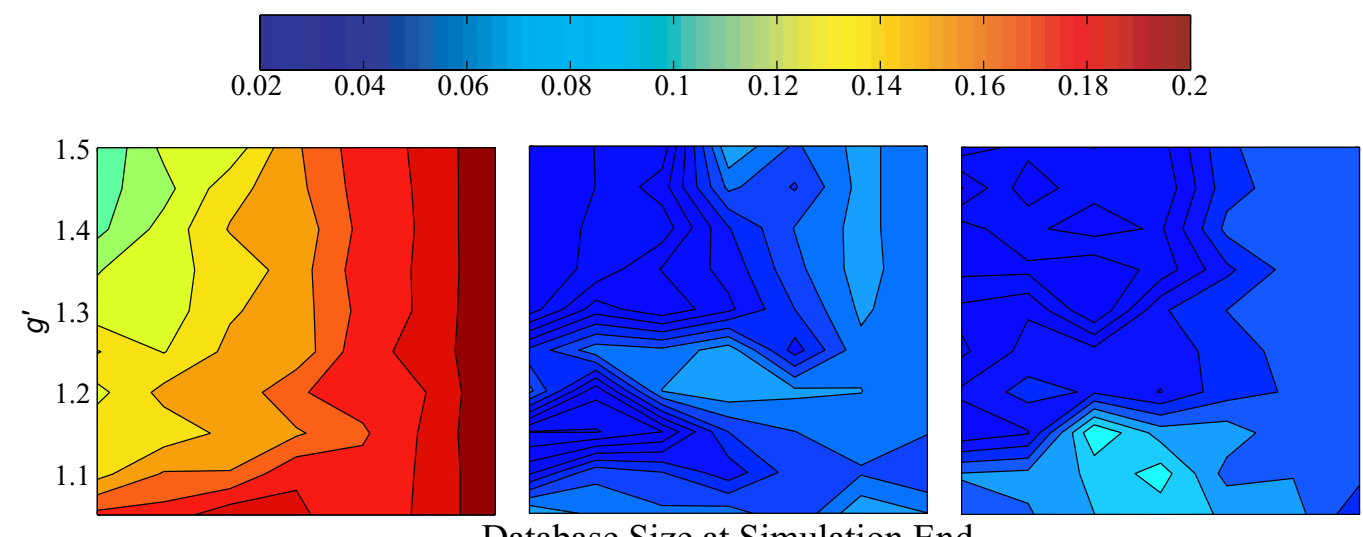

Database Size at Simulation End
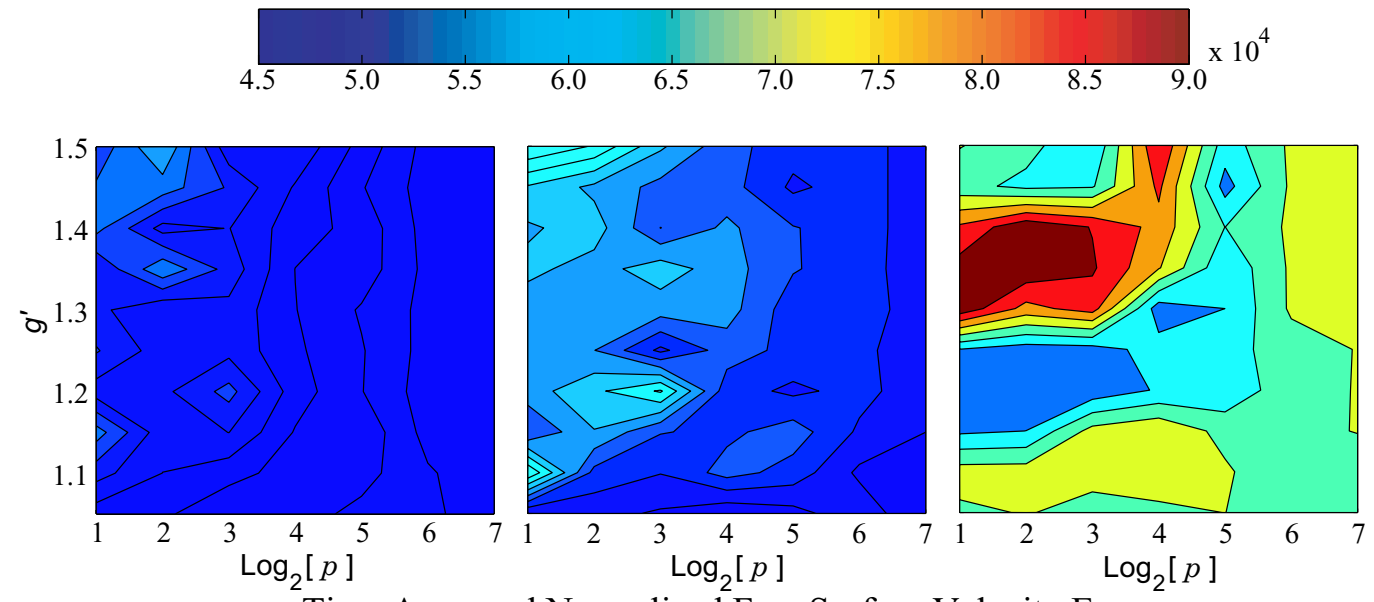

Time Averaged Normalized Free Surface Velocity Error

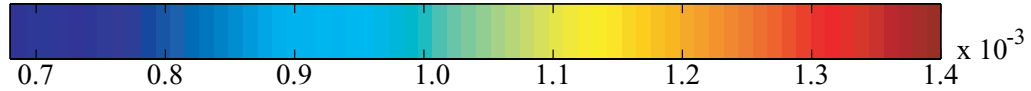

Fig. 6. Impact simulation results. 


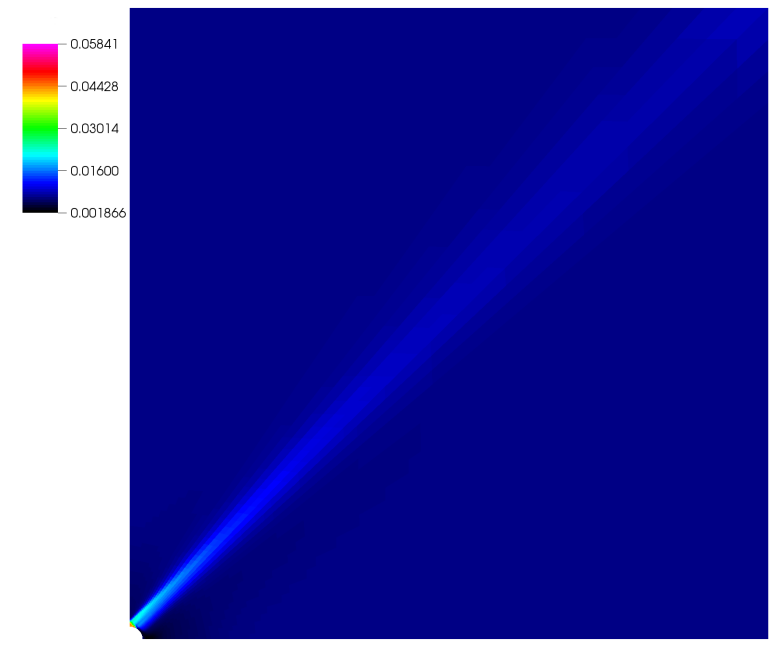

Fig. 7. Accumulated effective strain, 0.0019 to 0.058 .

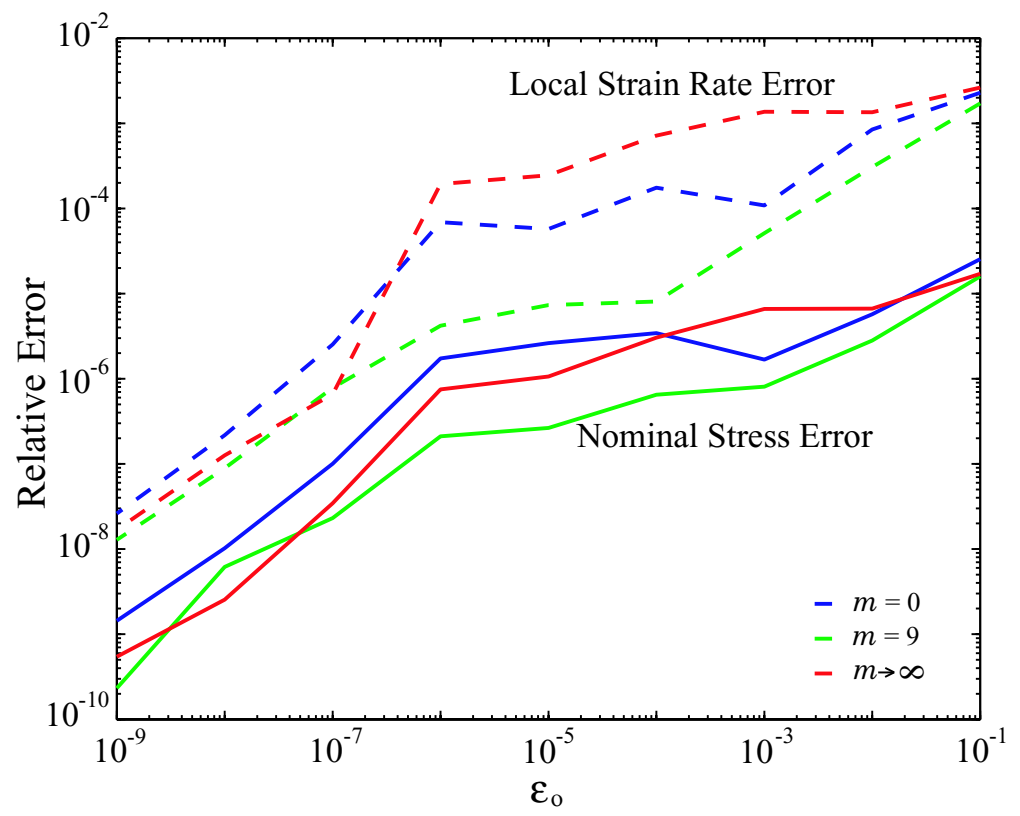

Fig. 8. Solution error as a function error tolerance in the adaptive tabulation strategy. Solid lines show the error in applied load and dashed lines show the strain rate error at a location near the stress concentration. 


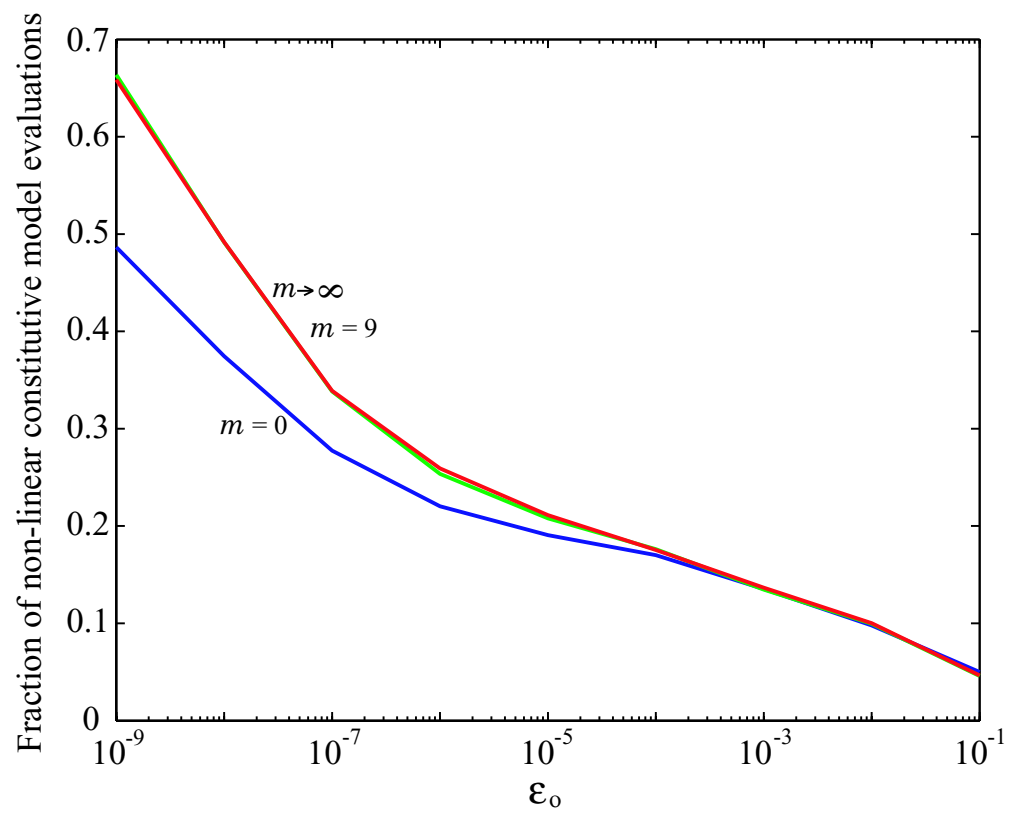

Fig. 9. Fraction of constitutive model evaluations as a function error tolerance in the adaptive tabulation strategy. 